\title{
ENCEFALOPATÍA NEONATAL DE ORIGEN INFLAMATORIO- INFECCIOSO. REPORTE DE CASO Y REVISIÓN DE LA LITERATURA
}

\author{
María del Pilar Medina-Alva ${ }^{1}$, Alvarado Merino Rosa Ysabel ${ }^{1}$, Ticona Vildoso Maricarmen², \\ Valeriano Valverde Eduar ${ }^{3}$.
}

\begin{abstract}
RESUMEN
La encefalopatía neonatal es la causa más común de atención neurológica en el recién nacido de término. Un porcentaje de los casos de encefalopatía se atribuye a eventos hipóxico-isquémicos intraparto, problemas metabólicos, accidentes cerebrovasculares y otras etiologías diversas, mientras que aproximadamente la tercera parte quedan sin una causa identificable.
\end{abstract}

La corioamnionitis y funisitis, procesos muchas veces silentes en la gestante, se han asociado desde hace muchos años a discapacidad en el niño, especialmente a parálisis cerebral, pero es más reciente la evidencia de que existe una respuesta inflamatoria fetal y encefalopatía aguda como proceso intermediario entre el mecanismo inflamatorio de la placenta y el cordón.

Se reporta un recién nacido de término con encefalopatía aguda grave de origen inflamatorio-infeccioso, demostrada por hallazgos placentarios, un cuadro clínico neurológico agudo y neuroimágenes compatibles.

Palabras clave: Encefalopatía neonatal, corioamnionitis, placenta (Fuente: DeCS BIREME).

\section{NEONATAL ENCEPHALOPATHY OF INFLAMMATORY-INFECTIVE ORIGIN. CASE REPORT AND REVIEW OF THE LITERATURE.}

\begin{abstract}
Neonatal encephalopathy is the most common cause of neurological consultation in the term newborn. Some cases of encephalopathy may be attributed to intrapartum hypoxic-ischemic events, metabolic alterations, stroke and other etiologies.

Chorioamnionitis and funisits are clinically silent in most pregnant women and have been associated for many years with disability, especially cerebral palsy. Recent data shows that systemic fetal inflammatory response and acute encephalopathy may be the intermediate processes between the inflammatory mechanism in the placenta and umbilical chord, and the occurrence of a cerebral lesion that produces disability.
\end{abstract}

We report the case of a term newborn with acute severe encephalopathy of inflammatory-infectious origin, demostrated with placental histopathology, and compatible clinical presentation and neuroimaging,

Key words: Neonatal encephalopathy, chorioamnionitis, placental (Source: MeSH NLM).

\section{INTRODUCCIÓN}

La encefalopatía neonatal (EN) se define como un síndrome de disfunción neurológica que afecta a recién nacidos mayores de 35 semanas, con una incidencia que se estima en 1-3/1000 nacidos vivos ${ }^{1}$. El cuadro clínico se caracteriza por deterioro del nivel de la conciencia, crisis epilépticas y dificultad en iniciar o mantener la respiración, que se acompañan de alteraciones en el tono muscular y los reflejos primitivos. La causa más reconocida de encefalopatía neonatal son los eventos hipóxico-isquémicos periparto (también llamados eventos centinela), los cuales sólo pueden identificarse en un $15-30 \%$ de los casos. Una serie de factores pueden estar implicados en el origen de la encefalopatía neonatal, por lo que la historia materna, los hallazgos de los exámenes auxiiares y los antecedentes obstétricos y factores anteparto e intraparto deben ser cuidadosamente evaluados para comprenderlo. Muchas observaciones recientes sugieren que la infección de las membranas y el cordón, denominadas corioamnionitis y funisitis, pueden ser causantes de encefalopatía neonatal y de la consiguiente lesión cerebral ${ }^{2,3}$. Reportamos el caso de un recién nacido con EN en quien se sospecha esta etiología de tipo inflamatorio-infeccioso.

Neuropediatra, Médico Asistente del Servicio de Neonatología del Instituto Nacional Materno-Perinatal, Lima, Perú

2 Médico Residente de la especialidad de Neurología Pediátrica, Universidad San Martín de Porres, Sede Hospital Nacional Edgardo Rebagliati Martins, Lima, Perú

Médico Residente de la especialidad de Neonatología, Universidad Antenor Orrego, Sede Hospital Víctor Lazarte Echegaray, Trujillo, Perú. Citar como: Medina-Alva P, Alvarado R, Ticona M, Valverde V. Encefalopatía neonatal de origen inflamatorio-infeccioso. Reporte de caso y revisión de la literatura. Rev Peru Investig Matern Perinat 2019; 8(2):40-4.

DOI: https://doi.org/10.33421/inmp.2019152

Recibido: 01-06-19 Aprobado: 28-06-19 


\section{REPORTE DE CASO}

El recién nacido fue de sexo masculino. Nació con Apgar $3-9$, con peso de 3,240 gramos, talla de $51 \mathrm{~cm}$, perímetro cefálico de $35 \mathrm{~cm}$. La madre tiene 33 años de edad, es según digesta con embarazo a término con historia de parto previo y no cursó con enfermedades durante la gestación. La serología es negativa. El embarazo fue controlado desde el primer trimestre de gestación con 9 controles prenatales. Inició trabajo de parto a las 41 semanas de gestación por fecha de última regla, el parto fue vaginal, con líquido amniótico meconial espeso. Durante la labor de parto se registró bradicardia fetal.

En las primeras horas de vida el recién nacido presentó dificultad respiratoria, siendo ingresado a la unidad de Cuidados Intermedios del Servicio de Neonatología. A las siete horas de vida presenta un evento de desaturación con cianosis que requirió ventilación a presión positiva e intubación endotraqueal siendo derivado a la Unidad de Cuidados Intensivos. A la exploración física inicial se encontraba con piel pálida, llenado capilar menor de dos segundos, tórax con retracciones subcostales e intercostales, pulmones con subcrépitos bilaterales, siendo el resto de la exploración normal. Después de su ingreso a $\mathrm{UCl}$, aún en el primer día de vida, se encontraba en ventilación mecánica con parámetros ventilatorios altos, lábil a la estimulación con tendencia a caída en la oximetría de pulso. La evaluación neurológica mostró un recién nacido con un gran compromiso neurológico, que se encontraba letárgico, con postura en flexión de extremidades. Al examen se encontró hipertonía global con normoreflexia, prensión palmar débil y reflejo plantar ausente, pupilas mióticas de $1 \mathrm{~mm}$ y poco reactivas. La fontanela se palpó tensa y llena.

Se planteó la posibilidad de una encefalopatía neonatal aguda, de etiologia a determinar. Se tomaron muestras

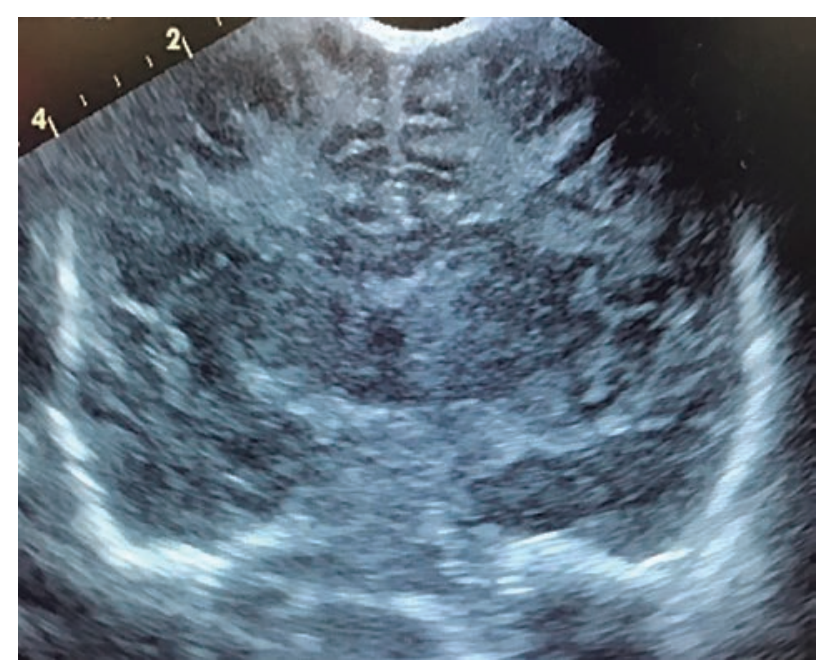

Figura 1. Ecografía cerebral del primer día de vida. Vista coronal. Se observa incremento de la ecogenicidad subcortical a nivel de ambos lóbulos frontales. para exámenes de laboratorio: Gasometria (después de la primera hora de vida) con acidosis mixta $(\mathrm{pH} 7.17$ $\left.\mathrm{PCO}_{2}: 50 \mathrm{HCO}_{3} 15 \mathrm{~EB}-1\right)$, hiperlactacidemia (6.6 mEq/L), electrolitos y hemograma normales, plaquetopenia moderada, proteína C reactiva incrementada $(17.9 \mathrm{mg} /$ $\mathrm{dL}$ ) y normoglicemia. Se tomaron hemocultivos y cultivo de líquido cefaloraquideo que resultaron negativos.

Se realizó ecografía cerebral que mostró hiperecogenicidad bilateral difusa de ambos hemisferios cerebrales con ventrículos colapsados. El estudio de flujometría doppler en las arterias cerebrales anteriores y pericallosas mostró incrementos en los índices de resistencia (figuras 1 y 2). Como parte de la evaluación de la encefalopatía aguda se colocó al paciente en monitorización contínua con electroencefalograma de amplitud integrada (aEEG), el cual mostró descargas compatibles con crisis epilépticas de varios minutos de duración que no presentaron correlato clínico (crisis electrográficas, figura 3). Se administró fenobarbital desde el primer día de vida, el cual se tituló por persistir con crisis eléctricas al segundo día de vida.

El paciente evolucionó en forma tórpida, se indicaron inotropos (dopamina y dobutamina) por signos de hipoperfusión tisular y cerebral, así como antibióticos por mala evolución clínica, proteína C reactiva alta y radiografía de tórax que persistían con infiltrados algodonosos.

Al sexto día de vida se le toma una resonancia magnética cerebral sin contraste con incremento de señal en T2 de la sustancia blanca frontal y occipital a predominio del hemisferio izquierdo. La corteza frontal y occipital bilateral también muestra áreas de alteración de señal hiperintensas en T1 con signos de restricción en la secuencia de difusión. Es de notar la intensa afectación del cuerpo calloso tanto en el cuerpo como en el esplenio (figuras 3 y 4 ).

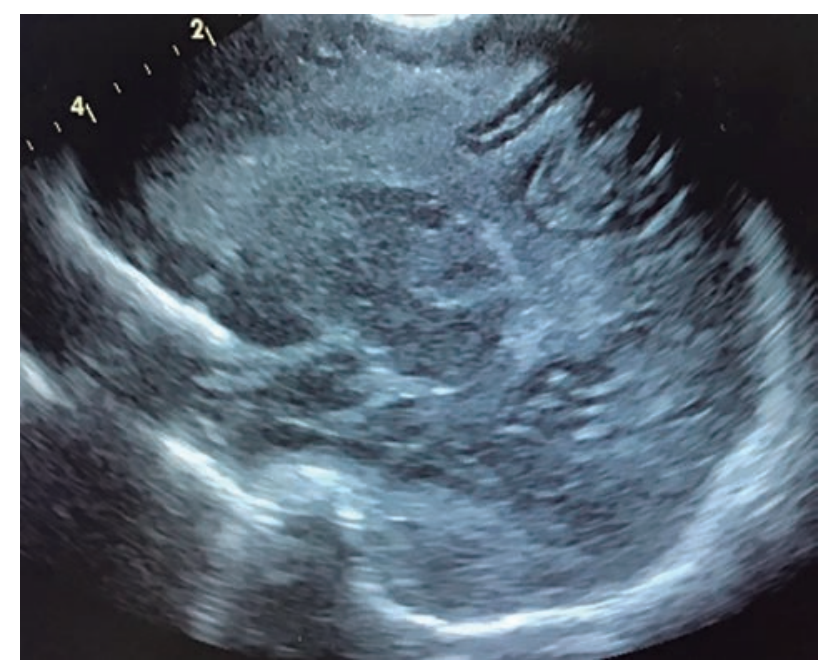

Figura 2. Ecografía cerebral del primer día de vida. Vista sagital derecha. Se observa incremento de la ecogenicidad subcortical a nivel de ambos lóbulos frontales y parietales 

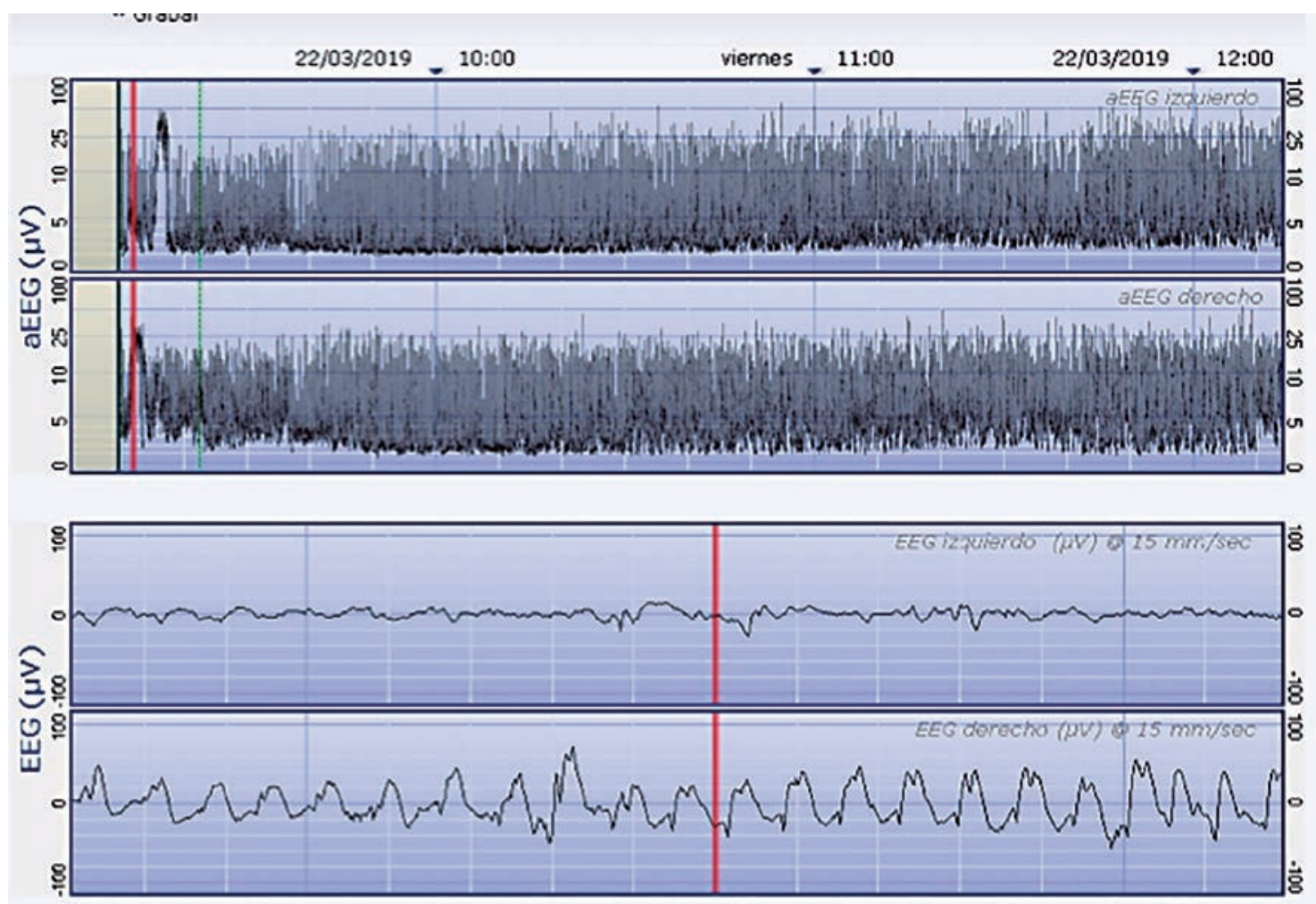

Figura 3a
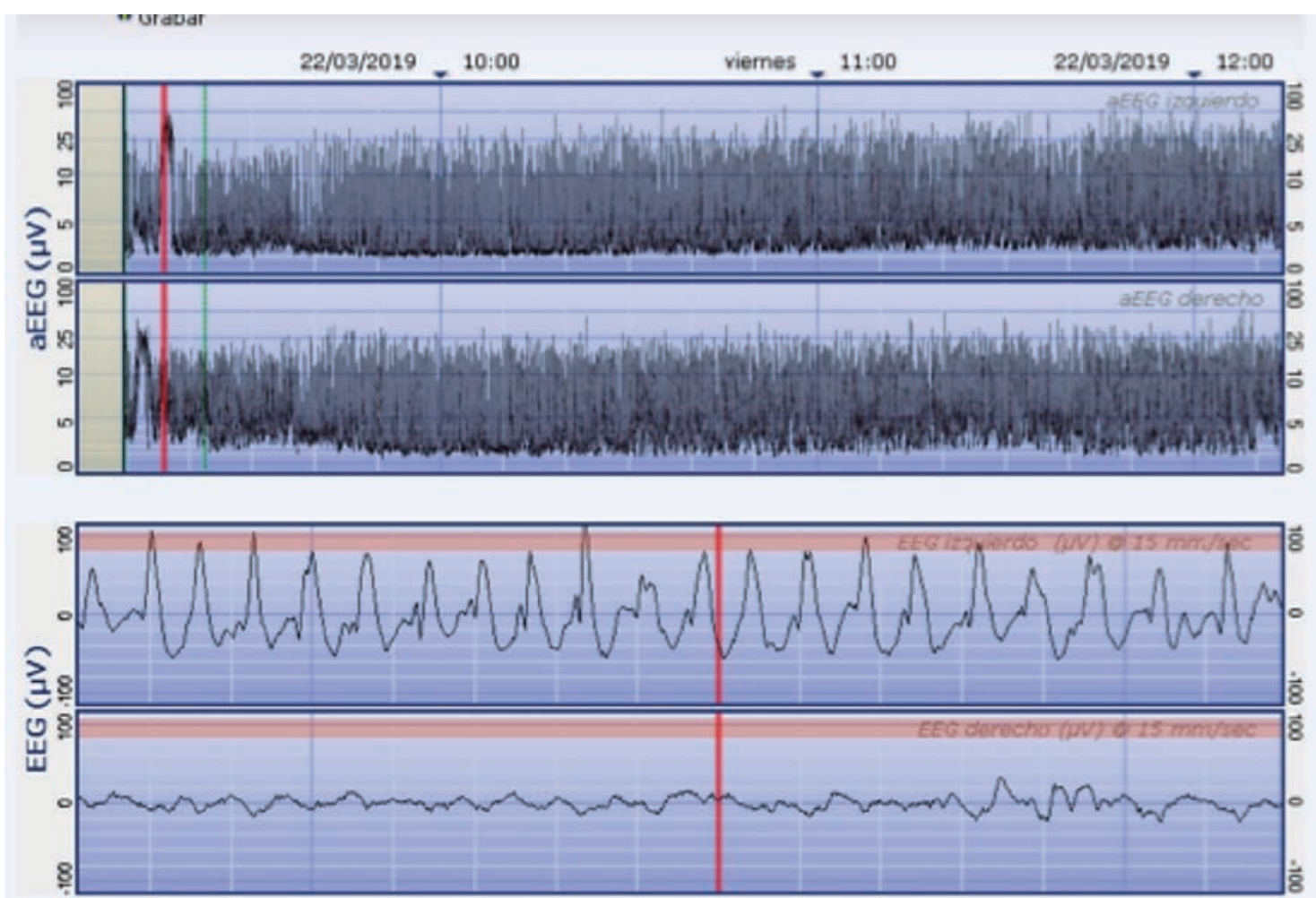

Figura 3b

Figura 3. Primeras 3 horas del registro aEEG. Se ha señalado las elevaciones del borde inferior del trazado primero en hemisferio derecho (3a) y después en el izquierdo (3b), que corresponden a crisis eléctricas en el trazado crudo. No se observaron crisis clínicas. En el registro continuo se hicieron evidentes otros períodos de crisis prolongadas que fueron consideradas como un estado epiléptico. 


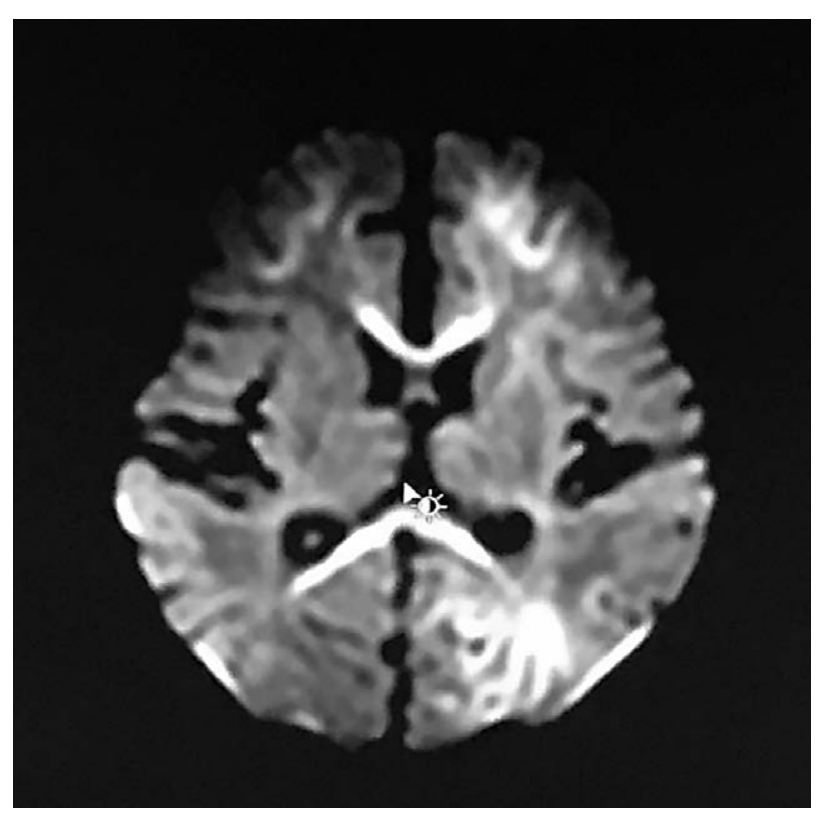

Figura 4a

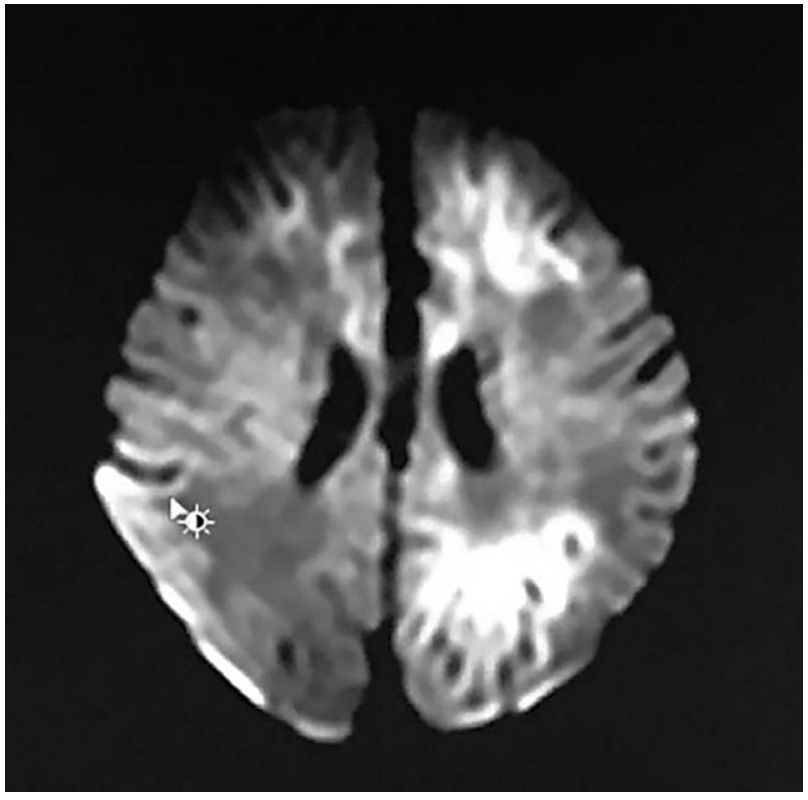

Figura 4b

Figura 4. RMN cerebral al sexto día de vida. Secuencia de difusión que muestra extenso compromiso del cuerpo calloso (4a) y de la sustancia blanca en patrón parasagital a predominio del hemisferio izquierdo (4b)

Se recibieron los resultados de patología de la placenta reportando corioamnionitis y funisitis aguda. El paciente ha evolucionado posteriormente en forma favorable, con mejoría progresiva de la encefalopatía y de la tolerancia oral, aun con antibióticos por una sepsis intrahospitalaria. Desde el punto de vista neurológico al alta se le encontró despierto, con hipertonía apendicular, reflejo palmar y plantar débiles, Moro completo y succión no sostenida. Logra fijar la mirada y seguir un objeto en blanco y negro, y se muestra atento a la voz.

El paciente empezó terapia física y orofacial antes del alta. Se normalizaron los hallazgos en la monitorización electrográfica por lo que se optó por el retiro de anticonvulsivantes. Ante los hallazgos de la resonancia y la evoluación clínica se espera que el paciente continúe su seguimiento en nuestra institución para poder determinar la extensión de las lesiones y el pronóstico a largo plazo.

\section{DISCUSIÓN}

La inflamación de la placenta, membranas y/o cordón umbilical es un antecedente común en los casos de depresión al nacer, puntajes bajos de Apgar, y encefalopatía neonatal en el recién nacido. La literatura médica muestra reportes que asocian la presencia de corioamnionitis y funisitis histopatológica con el hallazgo de discapacidad en el bebé a término, especialmente parálisis cerebral, con un riesgo estimado 4 veces mayor ${ }^{4}$. Sin embargo, en la población de prematuros, esta asociación no es consistente ${ }^{5}$. Existe evidencia de que la inflamación uteroplacentaria en animales de experimentación puede producir activación de la respuesta inflamatoria cerebral, traducida como activación de microglia, activación de macrófagos y muerte neuronal ${ }^{6}$. En el recién nacido de término, la presencia de señales inflamatorias procedentes de la placenta y/o membranas produce a su vez una respuesta inflamatoria fetal que puede acompañarse de encefalopatía aguda de diverso grado, que puede ser clínicamente similar a la producida por hipoxia-isquemia intraparto ${ }^{7}$.

La respuesta inflamatoria cerebral sería el proceso intermediario entre el mecanismo inflamatorio de la placenta y/o cordón y el origen de la lesión cerebral y puede ser demostrada en las neuroimágenes en la primera semana de vida. Esta afectación tiene predilección por la sustancia blanca cerebral ${ }^{8}$.

El mecanismo de lesión cerebral mediado por la infección placentaria es aún incierto. Se postula que (a) la elevada presencia de citokinas fetales puede causar daño directo al cerebro fetal (respuesta inflamatoria fetal), (b) que la inflamación de las membranas puede llevar a una interrupción del intercambio gaseoso, que resulta en injuria de tipo hipóxico-isquémico en el feto, (c) que la fiebre materna aumenta la temperatura central fetal, la cual a su vez es dañina para el cerebro en desarrollo, y (d) que la infección materna lleva a infección fetal directa del cerebro y las meninges (meningoencefalitis bacteriana), aunque esta afección es muy infrecuente ${ }^{4}$.

A medida que el feto llega al término, las zonas de perfusión limitada cambian desde el área periventricular a la corteza cerebral y los ganglios de la base. Cuando existe un evento centinela grave (asfixia aguda casi total), como en el caso del desprendimiento de la 
placenta o el prolapso de cordón, se produce injuria en las zonas metabólicamente más activas del cerebro, es decir, los tálamos, los ganglios basales y algunos núcleos del tronco cerebral, con el subsiguiente cuadro de encefalopatía neonatal evidente clínicamente. Cuando no existe dicho evento (asfixia parcial prolongada), la injuria ocurre en forma más silente, con un cuadro clínico menos obvio que sólo se manifiesta después del nacimiento y tiene una evolución variable ${ }^{9}$. En el caso de nuestro paciente, la encefalopatía fue mucho más manifiesta a partir del segundo día de vida, presentó crisis eléctricas que ameritaron tratamiento anticonvulsivante, y el patrón de lesión cerebral en RMN muestra afectación parasagital, sustancia blanca difusa y del cuerpo calloso, similar a lo descrito en eventos de asfixia parcial prolongada ${ }^{10}$.

Se han descrito alteraciones en el registro de EEGa en recien nacidos con antecedentes de corioamnionitis. La liberación de citoquinas proinflamatorias maternas y/o fetales, (especialmente IL-1 e IL-6) sería perjudicial para el sistema nervioso fetal en desarrollo, y su posible efecto se vería reflejado en cambios en la actividad eléctrica cerebral ${ }^{11}$.

No se han identificado biomarcadores que puedan diferenciar en forma específica la inflamación de origen infeccioso de la no-infecciosa. Se sospecha también que esta última puede producir lesión cerebral de diverso grado pero aún se trata de un área poco estudiada ${ }^{12}$

Shalak propone una vinculación causal entre la infección placentaria y un posterior cuadro de PC sólo en los casos en lo que se cumplan los siguientes criterios: evidencia de infección placentaria (histológica y/o clínica), mediadores inflamatorios elevados en el cordón umbilical y en la sangre del recién nacido, evidencia de encefalopatía moderada o severa y confirmación neurorradiológica de injuria cerebral reciente, todo esto en ausencia de acidemia cerebral severa ${ }^{13,14}$. Estas condiciones se cumplen en su totalidad en nuestro paciente, por lo que Concluimos que la causa de la injuria cerebral en este caso es de etiología inflamatoria infecciosa.

El estudio anatomopatológico de la placenta cobra entonces enorme importancia para el estudio de los recién nacidos con afectación neurológica temprana sin causa determinada ${ }^{15}$. Es importante la colaboración de los servicios (ginecoobstetricia, patología, laboratorio) para el correcto diagnóstico de estos pacientes.

Financiamiento: Autofinanciado.

Conflicto de interés: Los autores declaran no tener algún conflicto de intereses.

\section{REFERENCIAS BIBLIOGRÁFICAS}

1. Martinello K, Hart AR, Yap S, Mitra S, Robertson NJ. Management and investigation of neonatal encephalopathy: 2017 update. Arch Dis Child Fetal Neonatal Ed. 2017 Jul;102(4):F346-58.

2. Nelson KB, Penn AA. Is infection a factor in neonatal encephalopathy? Arch Dis Child - Fetal Neonatal Ed. 2015 Jan;100(1):F8-10.

3. Willoughby RE, Nelson KB. Chorioamnionitis and brain injury. Clin Perinatol. 2002;29(4):603-21.

4. Wu YW. Chorioamnionitis and Cerebral Palsy in Term and Near-Term Infants. JAMA. 2003 Nov 26;290(20):2677.

5. Shi Z, Ma L, Luo K, Bajaj M, Chawla S, Natarajan G, et al. Chorioamnionitis in the Development of Cerebral Palsy: A Meta-analysis and Systematic Review. Pediatrics. 2017 Jun;139(6):e20163781.

6. Hutton LC, Castillo-Melendez M, Smythe GA, Walker DW. Microglial activation, macrophage infiltration, and evidence of cell death in the fetal brain after uteroplacental administration of lipopolysaccharide in sheep in late gestation. Am J Obstet Gynecol. 2008 Jan;198(1):117.e1-117.e11.

7. Kim CJ, Romero R, Chaemsaithong P, Chaiyasit N, Yoon $\mathrm{BH}, \mathrm{Kim}$ YM. Acute chorioamnionitis and funisitis: definition, pathologic features, and clinical significance. Am J Obstet Gynecol. 2015 Oct;213(4 Suppl):S29-52.

8. Jenster M, Bonifacio SL, Ruel T, Rogers EE, Tam EW, Partridge JC, et al. Maternal or neonatal infection: association with neonatal encephalopathy outcomes. Pediatr Res. 2014 Jul;76(1):93-9.

9. Volpe JJ. Volpe's neurology of the newborn. Sixth edition. Philadelphia, PA: Elsevier; 2018. 1224 p.

10. Jin C, Londono I, Mallard C, Lodygensky GA. New means to assess neonatal inflammatory brain injury. J Neuroinflammation. 2015 Sep 25;12(1):180.

11. Paz-Levy D, Schreiber L, Erez O, Goshen S, Richardson J, Drunov Vi, et al. Inflammatory and vascular placental lesions are associated with neonatal amplitude integrated EEG recording in early premature neonates. Than NG, editor. PLOS ONE. 2017 Jun 23;12(6):e0179481.

12. Mandelbaum DE, Arsenault A, Stonestreet BS, Kostadinov S, de la Monte SM. Neuroinflammation-Related Encephalopathy in an Infant Born Preterm Following Exposure to Maternal Diabetic Ketoacidosis. J Pediatr. 2018 Jun;197:286-291.e2.

13. Shalak LF, Perlman JM. Infection markers and early signs of neonatal encephalopathy in the term infant. Ment Retard Dev Disabil Res Rev. 2002;8(1):14-9.

14. Meyer BA, Dickinson JE, Chambers C, Parisi VM. The effect of fetal sepsis on umbilical cord blood gases. Am J Obstet Gynecol. 1992 Feb;166(2):612-7.

15. Hayes BC, Cooley S, Donnelly J, Doherty E, Grehan A, Madigan $C$, et al. The placenta in infants $>36$ weeks gestation with neonatal encephalopathy: a case control study. Arch Dis Child - Fetal Neonatal Ed. 2013 May;98(3):F233-9.

Correspondencia: María del Pilar Medina-Alva

Av. Flora Tristán 194 Urb. Far West Vilas, La Molina, Lima- Perú.

Correo: mpilarmedina@gmail.com

Teléfono: 987709754 